\title{
Herpetiform pemphigus
}

INSERM

\section{Source}

INSERM. (1999). Orphanet: an online rare disease and orphan drug data base.

Herpetiform pemphigus. ORPHA:208524

Herpetiform pemphigus is a rare superficial pemphigus disease characterized by severe intractable pruritus with erythematous or urticarial plaques and vesicles organized in a herpetiform pattern. Mucosae are generally spared. Eosinophilia in peripheral blood and low titers of circulating autoantibodies are observed in many cases. Histologically, minimal or no apparent acantholysis is associated. 